\title{
Pharmacology and Forensics
}

Siniša Franjić*

Faculty of Medicine, Josip Juraj Strossmayer University of Osijek, Republic of Croatia

*Corresponding author: Siniša Franjić, Faculty of Medicine, Josip Juraj Strossmayer University of Osijek, Republic of Croatia

\section{Abstract}

Pharmacology is a science that studies the origins, physical and chemical properties, composition and action of drugs, fate in the body and elimination, as well as their therapeutic use. It includes pharmacodynamics and pharmacokinetics. Pharmacodynamics deals with the effect that a drug has on the body. It studies drug binding to receptors, including their sensitivity, postreceptor effects, and chemical interactions. Pharmacodynamics and pharmacokinetics help to understand the action of drugs. Physiological changes affect pharmacodynamics. The reasons are illness, age or the presence of other medications. Pharmacokinetics deals with the quantitative parameters of the fate of a drug in the body.

Keywords:Pharmacology; Forensics, Drugs; Intoxication

\section{Introduction}

One of the most common crimes in the United States is driving while under the influence of alcohol or drugs [1]. With every beer or cocktail a person consumes, alcohol seeps into the bloodstream. People who drink and drive carry the proof of their crime in their own veins. Blood alcohol content is the ratio of alcohol to blood in a person's body, and serologists can quickly determine this ratio with a simple blood test. If there is just one gram of alcohol for every five hundred grams of blood in the body, the blood alcohol content is 0.20 -more than twice the legal limit a driver is allowed to have (it is against the law in any state in the United States to drive with a blood alcohol content of more than 0.08 , and in some states, the limit is even lower). Detecting drunk drivers and proving their guilt is one of the quickest and most effective uses of forensic serology.

Whenever there is blood at the scene of a crime, it could be important proof that a victim or a suspect was at that particular place. In order for serologists to perform the series of tests on the blood that determine whether it is human, and if so, who it came from, the blood must first be identified and collected. This is not always a straightforward process. While some bodies are found lying in a pool of blood, at other crime scenes, blood is not so easy to find. Sometimes, a mere speck, drop, or smear suggests blood could be present. But blood, when it dries, turns dark brown and can be confused with other things, such as stains used to finish wood. Investigators may collect samples of any substance they find at the scene, but if the substance is not blood, it is not useful to a serologist.

\section{Cardiac Injury}

Litigation concerning claims of posttraumatic cardiac injury has been steadily increasing [2]. This increase has often been due to a presumption of injury or cardiac problem actually unrelated to an injury or, in fact, without substantial evidence for aggravation of the claimants' prior cardiac status. It is especially important in cases of suspected or proven myocardial contusion that the forensic medical specialist obtains as much previous medical history, information, and records regarding the presence of previous heart problems as possible.

Information conscientiously obtained and properly evaluated by an expert can be the determining factor in making or breaking a case that is presented for legal evaluation and subsequent judicial decision. As in all situations, the forensic medical specialist must be thoroughly knowledgeable, highly experienced, and as neutral and independent as possible. This is the basis upon which every case should be accepted and evaluated, for only then can honesty and justice be properly served.

The medical-legal implications of blunt chest trauma are horrendous in terms of cost and time, and all too often 
are plagued by erroneous or fallacious argumentation and reasoning. Careful clinical observation and skillful diagnostic ability combined with knowledge and experience are mandatory to attempt to resolve the complicated issues involved. A properly and carefully performed postmortem examination is helpful, but even under these circumstances the final decision can be difficult and insecure. One must take into account the type of injury, its location and extent, especially in relationship to the heart structures involved, and the possibility of myocardial pathology resulting from nontraumatic pathophysiology. Especially important are the implications of neurogenic stimulation and the resultant catecholamine release and shock as important factors in producing coronary vasospasm, plaque rupture, and cardiac dysrhythmia.

It cannot be overemphasized that whenever possible, one should obtain all of the available information regarding the historical presence of heart problems prior to the injury. These data should include electrocardiograms, chest x-rays, echocardiograms, ambulatory ECG reports, stress tests, heart scans, cardiac catheterization reports, hospital records, office records, and consultation records. Historical data relating to heart murmurs, cardiomegaly, symptoms of coronary insufficiency, cardiovascular drug therapy, and invasive or surgical intervention are especially important and, if present, are an obligatory requirement.

\section{Drug Consumption}

Both acute and chronic drug consumption induce thousands of deaths, producing a variety of autopsy findings depending primarily on the duration of intravenous drug consumption [3]. In addition to the consequences of the drug consumption itself, drug-associated diseases are often found, such as hepatitis and HIV, as well as other related infections (syringe abscesses, infected inguinal fistulas, thrombophlebitis, etc.). There are often drug-associated histopathological findings which, however, are not necessarily caused by the actual active ingredients in drugs. When immune status is impaired, immunological processes may also lead to histopathological findings in connection with other diseases (e.g., hepatitis, HIV, multiple syringe abscesses, injection of accompanying substances, etc.), all of which can also be observed independently of intravenous drug consumption. For this reason, this chapter is confined to clear and/or frequent drug-associated histopathological findings.

In the case of drug-induced lethal intoxication, massive pulmonary edema frequently weighing $>1000$ g per lung can be seen macroscopically. In cases of chronic and in particular intravenous drug consumption, histopathological findings are described, among others, in lung tissue, the heart, and the liver, as well as heroin-associated nephropathies (HAN). Substance-related histopathological findings are generally the result of chronic cocaine consumption with so-called cocaine cardiomyopathy and cocaineinduced organic infarction, as well as other complications. The fresh injection sites frequently found in drug-related deaths can be investigated immunohistochemically for the detection of morphine as well as for differentiation from insulin injection. Injected substances are transported lymphogenically and hematogenically.

\section{Drugs of Abuse}

The first indication that a death may be drug related is the observance of drug paraphernalia at the scene [4]. Syringes, needles, spoon "cookers" with small foil cooking sheets, lighters, bongs, etc., may be among the belongings of a drug abuser. Note the ice cream scooper that was used as a cooking utensil for heroin. It is not unusual to find components of a crack pipe in the pockets of an individual. The crack pipe is often recognizable as a hollow metal, glass, or plastic tube (often burned at one or both ends) along with a steel wool filter. The recognition of such drug paraphernalia is important and will help initiate appropriate toxicologic analyses. Although these items are highly suggestive of drug abuse, an individual's death may be of an entirely different nature. A complete autopsy is encouraged in such cases, because sometimes the death is actually determined to be of natural causes or some combination of natural, traumatic, and toxic reasons. One must also realize that drugs may not be consumed in the manner for which they were intended. For example, opioid pills (perhaps even the "timerelease" variety) may be ground down and injected intravenously, as may the film of drug from a fentanyl patch. These methods of drug abuse may provide a large bolus of medication over a short period of time that can prove fatal.

Emergency department physicians who request drug testing for a patient in their care should be aware of the strengths and limitations of the test methods available to them [5]. Consultation with a clinical toxicologist, a clinical chemist, or a clinical pathologist can help identify the likely drug(s) in question. Important variables that should be considered in regard to the assessment of a patient for drug use are sample collection, the type of test to be performed, and the types of substances that could interfere with the accuracy of the results. The most common samples for drug testing are urine and blood; hair and meconium can also be used.

Before concluding that "the tox screen is negative," the physician must know the methodology used by the 
laboratory. Does it perform comprehensive screens for multiple substances or single tests for specific xenobiotics? In addition, the physician should be aware of the test's detection and confirmation limits, especially for new drug analogs. Some drugs (e.g., ketamine) are difficult to detect with standard screening methods; referral to a reference laboratory might be required. Other drugs of abuse, especially amphetamines, are often modified in clandestine "laboratories," so it is difficult for medical facilities to remain current with analytic techniques and precautions against cross-reactivity.

For some substances, testing can indicate use of the drug but not necessarily intoxication with it. In an analysis for the presence of cocaine, for example, the screen detects benzoylecgonine, a metabolite formed soon after ingestion; therefore, a negative test excludes intoxication, within the appropriate detection levels in testing, but a positive test within the detection interval does not allow a conclusion to be made about if and when the patient was intoxicated. Detection intervals vary with manufacturers, and they can be influenced by the nature of the abuse (chronic vs. occasional). For interpretation of drug test results, physicians are encouraged to consult a medical toxicologist, who is specifically trained to interpret the patient's presentation in relation to the results of the analysis.

\section{Intoxication}

In forensic medical practice, toxin- and druginduced histopathological findings are primarily used to determine cause of death, but occasionally a plausible explanation of clinical symptoms can only be provided after a forensic chemical toxicological analysis [3]. Often, forensic autopsy yields nonspecific signs of intoxication, e.g., high-grade swelling of the brain and acute blood congestion of the viscera. Toxin-induced but in themselves nonspecific histopathological findings resulting from a reaction to legal or illegal substances are not uncommon, e.g., in the myocardium or in the striated skeletal musculature. Although numerous other substances can be readily determined in toxicological analysis, it is often the case that possible correlating histopathological findings cannot be causally and conclusively attributed to the ingested substances. Only in the case of sometimes patent overdoses is it possible to identify morphological findings in individual organs and attribute these to an active substance. For example, acetaminophen, an analgesic and antipyretic, can have hepatotoxic effects. Moreover, one must bear in mind that histopathologically detectable findings are not necessarily the result of the use of a single substance, but rather that only the combined use of two or more substances can cause pathological changes, e.g., if a relevant but (as yet) unknown genetic disposition is present. It is by no means the case that only ever synthetic substances are involvednaturally occurring substances can of course also have a toxic effect depending on the dosage of herbs or other plant constituents. A number of medications can cause (albeit it rarely or extremely rarely) severe toxic reactions involving the development of toxic agranulocytosis (e.g., carbimazole, a drug used for hyperthyroidism).

Poisoning is a pathophysiological condition resulting from the ingestion of toxic substances (poisons) and can be classified as either "chronic" or "acute" [6]. The clinical picture is referred to as toxicosis. Poisoning with more than one substance is referred to as mixed or polyintoxication. Patients often need to be treated symptomatically with invasive methods on the basis of mere suspicion, since methods of rapid toxicological analysis are generally lacking. However, invasive methods are mandatory, since in approximately $25 \%$ of all cases of poisoning, not the substance initially suspected but rather a completely different or even additional substance has been ingested. A recent report from a national poison information center indicates that the rate of lethal poisonings is equally distributed among adults and children, with a mortality rate of approximately $0.2 \%$ ( $1.5 \%$ according to other statistics). The report cites the circumstances of poisoning as accidental (65\%), suicidal (22\%), addiction-related (5.2\%), industrial ( $2.5 \%)$, and due to adverse drug (side) effects (1.8\%).

The significance of toxicological examinations of poisonous substances is illustrated by their rapid numerical and environmental growth, among which we count, besides the medications, herbicides and food additives used in everyday life, other injurious materials, for example industrial pollution [7]. The recognition, demonstration and quantification of these, among other things, constitute a part of the responsibility of toxicology.

The toxic material seriously injures or damages the vital functions of the organism, which is to say, it leads to death. With respect to their chemical natures, they could be such which damage various target organs (kidney, liver, hemopoetic organs), or have general effects. According to their mode of use they may be drugs, herbicides, solvents or food additives; according to their effects, carcinogens or mutagenic substances; according to their physical state, poisonous gasses, liquids, explosive materials, etc.; according to their biochemical effects, SH group inhibitors, a formers or influence of enzyme activities. Naturally, since any method of categorization is artificial, each toxin can belong to several groups due to its multiple effects. 
Medical complications of substance misuse may give an indication of a problem in the absence of acute symptoms or signs of intoxication [8]. Intravenous injection may result in superficial thrombophlebitis, deep vein thrombosis, and pulmonary embolus and chronic complications of limb swelling and venous ulcers. If injection occurs accidentally into an artery, vascular spasm may occur and result in ischemia, which, if prolonged, can lead to gangrene and amputation.

Cellulitis and abscesses may be seen around injection sites, and deep abscesses may extend into joints, producing septic arthritis. Self-neglect, malnutrition, and dental decay may occur, as may infectious diseases, such as hepatitis $B$ and $C$, human immunodeficiency virus (HIV), and the acquired immunodeficiency syndrome (AIDS).

Substance misuse is a major and growing problem often resulting in drug-related criminal activity [9]. Forensic physicians are seeing an increasing number of substance misusers in the setting of a police station. These misusers may be intoxicated, withdrawing or dependent on alcohol or drugs. The police may request a medical assessment of substance misusing detainees in order to: assess their fitness for detention and fitness to be interviewed; conduct a mental state examination; undertake a comprehensive examination of their fitness to drive a motor vehicle; and perform an intimate search for drugs.

Some substance misusers will, for a variety of reasons, attempt to hide their misuse from the authorities on arrest. Therefore, it is most important that examining forensic physicians make a conscious effort to look for any indication of substance misuse or dependence. A sympathetic approach from the doctor is more likely to result in disclosure and a reliable history from the detainee, who should be reassured that effective treatment will be given where necessary and that the overriding consideration of the doctor is their clinical safety and wellbeing.

\section{CSI}

A crime scene investigation begins with the initial response to a scene [10]. Securing and protecting the scene are important steps in a crime scene investigation, and this task is usually carried out by the first responding officer arriving on the scene. The entry of authorized personnel admitted to the scene should be documented using a log sheet. Suspects, witnesses, and living victims should be evacuated from the scene. If a victim is wounded, medical attention should be sought.

Appropriate supplies and devices should be used to prevent the contamination of evidence by investigators.
Protective wear and devices including a face mask or shield, safety eyeglasses, a disposable coverall bodysuit, gloves, shoe covers, and a hairnet should be used. Exposure to bodily fluids may occur during a crime scene investigation. An investigator can be exposed to bodily fluids through the mucous membranes, skin exposure, and needlestick injuries (especially when investigating a clandestine drug laboratory scene). Therefore, biosafety procedures must be followed for the protection of personnel from infectious bloodborne pathogens such as the human immunodeficiency virus (HIV), hepatitis B virus (HBV), and hepatitis C virus (HCV); infectious aerosol tuberculosis pathogens; and other biohazardous materials.

A preliminary survey should be carried out to evaluate potential evidence. In particular, the recognition of evidence plays a critical role in solving or prosecuting crimes. The priority of the potential evidence at crime scenes should be assessed based on each item's relevance to the solution of the case. Higher priority should be assigned to evidence with probative value to the case. For example, the evidence related to a corpus delicti is considered to be of the highest priority. Corpus delicti is a Latin term meaning "body of crime." In Western law, it primarily refers to the principle that in order for an individual to be convicted, it is necessary to prove the occurrence of the crime. In a forensic investigation, it also refers to the physical evidence proving that a crime was committed. For example, when an individual is missing, a missing persons investigation is usually initiated. If corpus delicti, such as a dead body or a victim's blood at a crime scene, is discovered during the investigation, a homicide case can be established and a suspect can be charged with homicide. Higher priority should also be attached to evidence that can establish connections such as victim-to-perpetrator linkage. For example, items found in a perpetrator's possession may be linked to a victim. This also applies to transfer evidence based on the principles of transfer theory, also known as the Locard exchange principle, which theorizes that the cross-transfer of evidence occurs when a perpetrator has any physical contact with an object or another person. Thus, trace evidence, such as hairs and fibers, may be transferred from a perpetrator to a victim or vice versa. This explains why it is important to ensure that perpetrators and their belongings are thoroughly searched for trace evidence. Likewise, victims and their belongings should be examined for the same reason.

\section{Forensics}

Pharmacogenetics and pharmacogenomics are gaining importance both in the clinical setting and in 
forensic pathology to investigate causes of death where no findings emerge from autopsy, and in the medical liability arena where scientific issues meet the justice system [11]. Generally speaking, pharmacogenetics is the study of how genetic variations give rise to differences in drug response, while pharmacogenomics (PGX) is the application of genomic technologies to the discovery of new therapeutic targets. Nevertheless, there is a diversity of opinion regarding the definitions and benefits of pharmacogenetics and pharmacogenomics. Depending on the purpose, pharmacogenetics can be used to define applications of single gene sequences or a limited set of multiple gene sequences, but not gene expression or genome-wide scans, to study variations in DNA sequences related to drug action and disposition. Pharmacogenomics can be used to define applications of genome-wide singlenucleotide polymorphism (SNP) scans and genome-wide gene expression analyses to study variations influencing drug action.

The first aspect of pharmacokinetics involves the entry of a drug into the body [12]. Chemicals, in the form of foods, medicines, drugs of abuse, or industrial chemicals, can enter the human body via several routes, including ingestion, inhalation, injection, skin application, and suppository. Except for cases of injection directly into the bloodstream, the chemical must pass through complex living cell membranes before it can enter the bloodstream.

For example, chemicals that enter the digestive tract must be absorbed by the cells lining the small intestine and then be transferred through the cells, where the chemical can then be absorbed by the capillary cells into the bloodstream. Chemicals that are inhaled must pass through the alveoli, the cells of the lungs, to get to the capillaries and enter the bloodstream. As chemicals pass into and out of cells, they must cross the cell membrane that keeps all of the cell contents securely inside, but which allows some materials to pass. Chemicals can move through the cell membrane through one of several mechanisms.

Pharmacodynamics is the study of the mechanisms of drug action. How does a chemical cure disease, stimulate or inhibit the nervous system, change behavior, influence our digestive system, or induce a toxic reaction? The body itself is made up of chemicals, and when drugs (chemicals) are taken, the drugs interact with the body's chemicals and these interactions result in biochemical and physiological effects. While there are many different mechanisms of drug action that account for the different effects of diverse drugs, in this book we will restrict our discussion to those reactions that explain the effects of drugs of abuse.
Drug- and toxin-related deaths represent a sizeable percentage of cases investigated by death investigation offices [13]. Frequently in these cases, there is no obvious visible indication of drug or toxin exposure, either on external autopsy examination or internal examination (grossly or microscopically). In a large number of such cases, there may also be no scene indicators that suggest drugs (this may or may not be due to the fact that family members or friends might have "cleaned-up" the scene). Since most drug-related deaths have no definitive external or internal findings, these deaths are included in the larger population of deaths that have "no anatomic cause of death." Other case types that may be included in this population are drownings, certain electrocutions, temperature-related cases, seizure disorders, and deaths from cardiac conduction system disorders. Drug deaths may involve illegal ("street") drugs, over-the-counter (OTC) preparations, or prescription medications. In order to appropriately identify deaths related to drugs or toxins, it is essential to perform forensic toxicology tests on postmortem blood (and/or other tissue) samples. For this reason, forensic pathologists and death investigators should be very familiar with the types of drugs that are routinely screened for by their forensic toxicology laboratory. Routine "drug screens" usually identify many drugs and toxins; however, for many others, the toxicology laboratory must be asked to specifically attempt to identify that particular drug or toxin. In order to know which ones might be present, a thorough death and scene investigation must be performed. Slang or street names are very common for drugs of abuse. It is beyond the scope of this text to list each of these. The reader is referred to several websites to view such listings.

\section{Autopsies}

There are essentially two types of autopsies-the clinical or academic autopsies done at hospitals and the forensic autopsies executed in the medicolegal settings [14]. The aim of the clinical autopsy is to find out, clarify, or confirm diagnoses that remained unknown or are not sufficiently clear during the stay of a patient in a hospital or health institution. The forensic autopsy is performed under the supervision of a legal authority such as a prosecutor, a procurator fiscal, a magistrate, a judge, a coroner, medical examiner, or the police. The forensic or medicolegal autopsy primarily focuses on violent deaths (accidents, suicides, and homicides), although in many situations, it also deals with natural deaths that should be the object of a clinical autopsy. Suspicious and sudden deaths, deaths without medical assistance, and deaths that are litigious or related to surgical or anesthetic procedures, must also to be clarified by a forensic autopsy. 
Usually in the majority of jurisdictions, relatives' permission is not necessary to carry out the autopsy. Eventual obstruction of justice is then avoided. In some regions, the forensic autopsies are divided in two categories - criminal deaths (suspicious or because of murder, homicidal suffocation or smothering, infanticide) and noncriminal deaths (suicides, natural deaths, accidents, etc.). Many people, including some specialists working in the area, have difficulties in distinguishing among cause, manner, and mechanism of death. Cause of death means any injury or disease that generates a pathological alteration in the body that leads to the individual's death. Examples are esophageal carcinoma, a myocardial infarction, blunt trauma of the head, or a gunshot wound of the thorax.

The mechanism of death signifies the pathological alteration resulting from the cause of death: hemorrhage, infection, fatal arrhythmia. It is possible that a mechanism of death is shared by different causes of death: a hemorrhage may result from blunt trauma, stabbing, or lung carcinoma. The contrary is also true, as the cause of death can give origin to different mechanisms of death: a gunshot wound can produce a hemorrhage, but if the victim had survived, an infectious complication is the mechanism.

The manner of death (known in Latin countries as "medicolegal etiology," where it is considered as another objective of the autopsy) means, in violent deaths, the distinction among an accident, suicide, homicide, or an undetermined death. Following the above-mentioned way of thinking, a manner of death can be the result of multiple causes and mechanisms of death. The classic example is the one of a gunshot injury (cause of death), which could be classified as the four manners of death: homicide (someone shot the victim), suicide (the shot was self-inflicted), accident (the shot was selfinflicted unintentionally), and undetermined (there are no witnesses to the events, and the autopsy failed to clarify the manner of death). Mechanism of death could be hypovolemic shock by hemorrhage or, if the person survived, a thromboembolic or infectious complication, such as a bronchopneumonia or peritonitis.

The recognition and collection of evidence is an important part of forensic pathology practice and distinguishes it from a hospital-consent autopsy [15]. Toxicological analysis assists in the determination of the cause of death in certain cases (e.g., suspected medication or illicit drug overdose) when there is "no anatomic cause of death," i.e., no gross or microscopic cause of death. Toxicology samples-most commonly blood and urine-are collected. To ensure continuity of evidence, sample tubes and containers are sealed. The seal label indicates the name of the deceased, case number, date and time sealed, and the type of specimen, and is signed by the pathologist or police officer attending the autopsy. If an injured individual survives in the hospital for a period of time and then dies, any ethanol or other drugs will have metabolized, rendering postmortem specimens useless for meaningful analysis. Admission blood samples, if available, need to be obtained. The laboratory must be informed to hold these samples either by a coroner/medical examiner or designate (pathologist, police officer). In this situation, the hospital needs to have a tracking system to ensure continuity. If samples do not require analysis, they can be stored.

\section{Conclusion}

Drugs are selected according to their properties and according to the characteristics of the patient. The riskbenefit ratio is also assessed; each drug also brings certain dangers. The response to the prescribed medication also depends on the nature of the patient, the accompanying diseases and taking other medications. The therapeutic response is also affected by professional errors. If the existence of a criminal offense in the procedure of giving a medicine to a patient is established, then a criminal liability procedure is initiated to determine who is responsible for it. The answers to these questions will be given by forensic experts, ie experts in pharmacological forensics. Otherwise, forensics is the name for the application of a wide range of scientific branches to establish facts in court proceedings. Forensic scientists collect, store, and analyze scientific evidence during an investigation. While some forensic scientists travel to the crime scene to gather evidence themselves, others take on a laboratory role, performing analyzes on cases brought to them by other individuals. In addition to their laboratory role, forensic scientists testify as experts in court proceedings and can work for either the prosecution or the defense. Although any area could technically be forensic, certain parts have evolved over time to cover most forensically related cases.

\section{References}

1. MacKay J (2009) Forensic Biology. Lucent Books, Gale, Cengage Learning, Detroit, USA p. 58-59.

2. RuDusky B (2009) Forensic Cardiovascular Medicine. CRC Press, Taylor \& Francis Group, Boca Raton, USA p. 11-12.

3. Dettmeyer RB (2018) Forensic Histopathology - Fundamentals and Perspectives, Second Edition. Springer International Publishing AG, Cham, Switzerland 85: 123.

4. Dolinak D (2005) Toxicology in Dolinak D, Matshes EW, Lew EO (Eds.), Forensic Pathology - Principles and Practice. Elsevier, Academic Press, Burlington, USA, pp. 492.

5. Dyer KS (2007) New Drugs of Abuse in Olshaker, J. S.; Jackson, M. C.; Smock, W. S. (eds): Forensic Emergency Medicine, Second Edition. Lippincott Williams \& Wilkins, Wolters 
Kluwer, Philadelphia, USA, pp. 224.

6. Dettmeyer RB, Verhoff, MA, Schütz HF (2014) Forensic Medicine - Fundamentals and Perspectives. Springer-Verlag Berlin Heidelberg, Berlin, Germany, pp. 495-496.

7. Buris L (1993) Forensic Medicine. Springer-Verlag, Budapest, Hungary, pp. 313.

8. Stark MM, Norfolk G (2005) Substance Misuse in Stark, M. M. (ed): Clinical Forensic Medicine, Second Edition - A Physician's Guide. Humana Press Inc., Totowa, USA, pp. 289.

9. Stark MM, Norfolk GA (2009) Substance misuse in McLay, W. D. S. (ed): „Clinical Forensic Medicine, Third Edition. Cambridge University Press, Cambridge, UK, pp. 79.

10. Li R (2015) Forensic Biology, Second Edition CRC Press, Taylor \& Francis Group, Boca Raton, USA, p. 3-6.

11. Pelotti S, Bini C (2011) Forensic Pharmacogenetics in Vieira,
D. N. (ed): Forensic Medicine-From Old Problems to New Challenges. InTech, Rijeka, Croatia, pp. 267.

12. Zedeck BE, Zedeck MS (2007) Forensic Pharmacology. Chelsea House, Infobase Publishing, New York, USA, pp. 12-18.

13. Prahlow J (2010) Forensic Pathology for Police, Death Investigators, Attorneys and Forensic Scientists. Springer Science+Business Media, LLC, New York, USA, pp. 257-258.

14. Pinheiro, J (2006) Introduction to Forensic Medicine and Pathology in Schmitt A, Cunha E, Pinheiro J (Eds.), Forensic Anthropology and Medicine - Complementary Sciences from Recovery to Cause of Death. Humana Press Inc, Totowa, USA, p. 16-19.

15. Shkrum MJ, Ramsay DA (2007) Forensic Pathology of Trauma - Common Problems for the Pathologist. Humana Press Inc, Totowa, USA, p. 15.

*Corresponding author: Siniša Franjić, E-mail: sinisa.franjic@gmail.com

Next Submission with BGSR follows:

- Rapid Peer Review

- Reprints for Original Copy

- E-Prints Availability

- Below URL for auxiliary Submission Link: https://biogenericpublishers.com/submit-manuscript/ 